\title{
BMJ Open The Belgian policy of funding antimicrobial stewardship in hospitals and trends of selected quality indicators for antimicrobial use, 1999-2010: a longitudinal study
}

Marie-Laurence Lambert, ${ }^{1}$ Robin Bruyndonckx, ${ }^{2}$ Herman Goossens, ${ }^{3}$ Niel Hens, ${ }^{2,4}$ Marc Aerts, ${ }^{2}$ Boudewijn Catry, ${ }^{1}$ Fiona Neely, ${ }^{1}$ Dirk Vogelaers, ${ }^{5}$ Naima Hammami ${ }^{1}$

To cite: Lambert M-L, Bruyndonckx R, Goossens $\mathrm{H}$, et al. The Belgian policy of funding antimicrobial stewardship in hospitals and trends of selected quality indicators for antimicrobial use, 1999-2010: a longitudinal study. BMJ Open 2015;5:e006916. doi:10.1136/bmjopen-2014006916

- Prepublication history for this paper is available online. To view these files please visit the journal online (http://dx.doi.org/10.1136/ bmjopen-2014-006916).

Received 14 October 2014 Revised 16 January 2015 Accepted 19 January 2015

CrossMark

For numbered affiliations see end of article.

Correspondenceto Dr Marie-Laurence Lambert; mllambert@wiv-isp.be

\section{ABSTRACT}

Objectives: In order to improve antimicrobial (AM) use, a policy of providing technical and financial support to AM management teams (AMTs) was rolled out in all Belgian hospitals between 2002 and 2008. We aimed to analyse the association of this policy with AM use for the two indications accounting for the largest number of patients receiving AM: prophylaxis for major lower limb orthopaedic surgery and pneumonia.

Design, setting, participants: We used patient-level data routinely collected in all Belgian acute care hospitals between 1999 and 2010. We modelled trends for selected quality indicators (QIs) using the year of AMT implementation in each hospital as the main 'change point', with fine-tuned case-mix adjustment. Of all admissions for lower limb orthopaedic surgery, and pneumonia between 1999 and 2010, 90\% (325 094) and $95 \%$ (327 635), respectively, were found eligible for analyses.

Outcomes: The surgery QI was defined as: cefazolin, dose in the expected range, and no use of other AM. For pneumonia, Qls were: ratio of oral/parenteral defined daily doses (DDD, O/P QI), and mean number of DDD minus penicillin, per 100 days of hospitalisation (DDD QI).

Results: Between 1999 and 2010, the surgery QI improved from $59 \%$ to $71 \%$, the $\mathrm{O} / \mathrm{P} \mathrm{Q}$ I from 0.72 to 0.97 , and the DDD QI from 96 to 64 . Heterogeneity between hospitals was high. Overall, no association was found with the year of implementation of the AMT.

Conclusions: Improvements have been observed but could not be related at the national level to the policy under study. However, these results cannot be extrapolated to other Qls for AM use in hospitals. Our findings do not question the need for AMT, nor the need for continuation of AMT funding. Several recommendations can be made in order to make the best of Belgium's unique political and financial commitments in that field.

\section{INTRODUCTION}

The inappropriate use of antimicrobials (AM) and its consequences in terms of infections due to AM-resistant microorganisms
Strengths and limitations of this study

- First study to evaluate a national policy of implementing and financing antimicrobial management teams in hospitals with a longitudinal study design.

- Exhaustive database on all admissions in Belgian hospitals over 11 years for the indications accounting for the largest number of patients receiving antimicrobials in hospitals (antimicrobial prophylaxis for lower limb orthopaedic surgery, pneumonia).

- Fine-tuned case-mix adjustment when comparing hospitals before/after, and with/without the policy, taking into account the possible time lag between implementation and results.

- Quality indicators selected for this study measure some important aspects of quality, but fall short of providing a global picture of overall quality of antimicrobial use in hospitals. This is intrinsically complex, and other indicators might provide different results.

- This study has shown no overall impact of the policy on national trends, but has not investigated the factors that determine success or failure at hospital level.

represents a major public health problem. ${ }^{1}$ Although the largest part of AM consumption takes place in the community, ${ }^{2}$ the risks are concentrated in hospitals: $35 \%$ of hospitalised patients in Europe $^{3}$ and $52 \%$ in the $\mathrm{USA}^{4}$ are taking an AM. Over one-third of prescriptions might not be compliant with evidence-based guidelines. ${ }^{5} \mathrm{AM}$ stewardship, conducted by AM management teams (AMTs), is a key strategy to improve AM prescribing, to ensure effective treatment of patients and to reduce AM resistance in hospitals. ${ }^{6}$ The need for a legal basis, regulatory mechanisms and funding for AM stewardship programmes and research are widely endorsed. ${ }^{78}$

Belgium has been a pioneer in this field. The Belgian Antibiotic Policy Coordination 
Committee (BAPCOC) was created in 1999 in order to improve $\mathrm{AM}$ use in Belgium and has been very active since then at different levels. ${ }^{9}$ Since July 2007, all acute care hospitals in the country have been receiving financial and technical support for hiring a trained manager for their AMT; the initiative was first piloted in 37 voluntary hospitals (2002), then extended to another 24 hospitals (2006), and finally to the remaining hospitals (55) in 2007. Apart from funding, the intervention included technical guidance and advanced specialist training for the formal establishment and follow-up of AMTs. However, hospitals were not given targets, and were left to devise their own interventions and themes. A selfreporting survey was conducted in 2007. Antibiotic stewardship tools used by AMTs included (non-exhaustive list): an antibiotic formulary (96\% of hospitals), practice guidelines for antibiotic therapy and surgical prophylaxis (92\%, and $96 \%$, respectively), a list of restricted AM agents $(76 \%)$, sequential intravenous/oral therapy with equivalent bioavailability $(79 \%) .{ }^{10}$ The minimum composition, mandate and tasks of hospital AMT have a legal basis since 2008. The largest part of the BAPCOC budget now goes to staffing and technical support for AMT in all Belgian acute hospitals. ${ }^{9}$ To the best of our knowledge, Belgium has been the first country to provide hospitals with structural, earmarked funding for AMT, making the experience a test case for such a policy.

The objectives of our study were to analyse the association of this policy with trends of selected quality indicators (QIs) for AM use in hospitals. We focused on the indications accounting for the largest number of patients receiving AM.

\section{METHODS}

\section{Source of data and Qls}

We obtained patient-level data routinely collected between 1999 and 2010 in all Belgian acute care hospitals for pathology-based financing purposes. These include the all-patient-refined diagnosis-related group (APR-DRG, V.15) and associated severity of illness (SoI, $1-4)^{11}$ as well as details on each AM used during the stay (molecule, dose and route of administration); however, the timing of AM administration is lacking. The SoI for the hospital stay is assigned following a complex computerised algorithm that takes into account the patient's primary and secondary diagnoses, procedures and age (the underlying clinical principle is that patients with a high SoI are characterised by multiple serious diseases or illnesses). ${ }^{11}$

We used the WHO anatomical therapeutic chemical (ATC) /defined daily doses (DDD) method, ${ }^{12}$ applying version WHO 2010 to all 12 years in the database. Comparing hospitals (with or without the policy, as well as before and after implementation of the policy) was done per APR-DRG to facilitate case-mix adjustment. The two APR-DRG with the highest number of patients receiving AM were APR DRG 302 (major lower limb orthopaedic surgery without trauma-mainly hip or knee replacement—or revision) and APR DRG 139 (simple pneumonia). We decided against clinical outcome measures such as mortality, readmission and length of stay (LoS) because these are too indirectly related to appropriate AM prescribing. Compliance with guidelines for antibioprophylaxis in APR DRG 302 ('surgery QI') was defined using three criteria at patient level as: (1) choice of AM (cefazolin), dose in the expected range (2-8g) and (2) no other AM given during the stay. ${ }^{13}$ We excluded from the analyses hospital stays with secondary infectious diagnoses and SoI 3 and 4, so that it could fairly be assumed that there was no other reason for prescribing an AM than antibioprophylaxis. For pneumonia, the data did not allow assessing the appropriateness of treatment at patient level. In addition, treatment guidelines can differ between hospitals. Aggregating data at hospital level, we selected two QIs: (1) the ratio of oral DDD/parenteral DDD (' $\mathrm{O} / \mathrm{P}$ QI'), as early parenteral to oral switch is recommended to reduce $\mathrm{LoS}$, adverse events related to intravenous line, and costs ${ }^{13-15}$ and (2) total DDD (J01) minus penicillin (J01C), per 100 days in hospital for pneumonia ('DDD QI'), under the assumption 'less is better'. Penicillin use was excluded because recommended daily doses have increased over the years.

\section{Statistical approach and adjustment for confounding}

We used a change point model. ${ }^{16}$ Such a model tests whether the slope of a trend is different before and after the change point. We included as change points the year each hospital received funding for its AMT (2002, 2006, 2007), as well as the years 2001 (the year of a very large awareness campaign for AM) and-for pneumonia only-2006 (when a new financing mechanism for hospital drugs was introduced; however, this mechanism did not apply to AM prophylaxis). We then tested the need to include additional change points. The surgery QI was a dichotomous variable at the patient (stay) level, and variables adjusted for were LoS, age (continuous), gender, SoI (1-2), and intensive care unit (ICU) stay (at some point, hospitalised in the ICU). For pneumonia, outcomes were continuous variables at hospital level and required aggregation of patient (stay) level data. Hospital variables for pneumonia stays were: median LoS, distribution of the stays (\%) according to SoI (1-4), gender, ICU stay, patient origin at admission (home, long-term care facility, other or unknown), discharge status (dead/alive), and age categories $(<1,1-5$, $6-10,11-16,>16$ years). Caseload (number of hospital stays included in the analyses for each QI, per hospital, and per year) was also included in the model as a variable at hospital level.

For pneumonia, results were weighted for caseload. A time lag $( \pm 1$ year $)$ was introduced to account for the fact that the effect of AMT funding might have started before the hospitals received the money (eg, because 
preparation included AM stewardship training) and might need some time to be fully implemented (eg, because of possible delays in recruiting an AM specialist).

\section{Statistical model and software}

A somewhat abrupt but continuous change in the evolution of the outcome over time can be modelled by including a change point in the generalised linear mixed model (GLMM). ${ }^{17}$ A GLMM is an extension of a generalised linear model in which the correlation between measurements from the same hospital is taken into account using so-called random effects. Whereas the outcome is considered to belong to the exponential family (including the normal and the Bernoulli distribution), the systematic component of a GLMM is given by:

$$
\begin{aligned}
\mathrm{g}\left(\mu_{\mathrm{ij}}\right)= & \left(\beta_{0}+\mathrm{b}_{0 \mathrm{i}}\right)+\left(\beta_{1}+\mathrm{b}_{1 \mathrm{i}}\right) \mathrm{t}_{\mathrm{ij}}+\beta_{2}\left(\mathrm{t}_{\mathrm{ij}}-\mathrm{CP}\right)_{+} \\
& +\sum_{\mathrm{q}=3}^{\mathrm{Q}} \beta_{\mathrm{q}} \mathrm{X}_{\mathrm{qij}}
\end{aligned}
$$

where $\mu_{\mathrm{ij}}$ is the average outcome for hospital $\mathrm{i}(\mathrm{i}=1,2, \ldots$, $\mathrm{N})$ at time points $t_{i j}\left(j=1,2, \ldots, n_{i}\right), g($.$) is a link function$ (eg, an identity link or a logit link), $\mathrm{N}$ is the total number of hospitals, $n_{i}$ is the number of observations from the $\mathrm{i}$-th hospital, $\mathrm{t}_{\mathrm{ij}}=1$ corresponds to the start of the study, $\beta_{0}$ is the fixed intercept, $b_{0 \mathrm{i}}$ is the hospitalspecific deviation from intercept $\beta_{0}, \beta_{1}$ is the fixed slope, $\mathrm{b}_{1 \mathrm{i}}$ is the hospital-specific deviation from slope $\beta_{1}$, $\mathrm{x}_{+}=\max (\mathrm{x}, 0)$ such that $\beta_{2}$ is the global difference in the linear trend before and after the change point, CP is a global change point, $\beta_{\mathrm{q}}(\mathrm{q}=3,4, \ldots, \mathrm{Q})$ are the fixed effects for the explanatory variables $\mathrm{X}_{\mathrm{qij}}$ and $\mathrm{Q}$ is the number of explanatory variables. All models were fitted using SAS V.9.3.

\section{RESULTS}

\section{Surgery QI}

A total of 325094 hospitals stays between 1999 and 2010 were available for analyses $(90 \%$ of the total number of stays coded APR DRG 302, after exclusion of SoI 3 and 4 , stays with infectious diagnoses, and errors in hospital coding). Median LoS in this population decreased from 15 days in 1999 to 8 days in 2010. Trends in the number of hospital stays and compliance with the three criteria, as defined earlier, are shown in table 1. Compliance increased from $53 \%$ of included stays in 1999 to $71 \%$ in 2010 .

Based on likelihood ratio (LR) tests comparing models with and without specific change points, the change point 'year of AMT' was retained in the model $(\mathrm{p}<0.0001)$; the change point 'year 2001' was identified as statistically significant $(p<0.0001)$. All changes were negative and weak (meaning that compliance increased, but at a slower pace than before). The final model had a pseudo- $\mathrm{R}^{2}$ value of 0.1927 . Although $\mathrm{R}^{2}$ values for models on binary outcomes are typically much lower than for models on continuous outcomes, this indicates
Table 1 Hospital stays for lower limb orthopaedic surgery ${ }^{\star}$ and compliance with guidelines for surgical

\begin{tabular}{|c|c|c|c|c|}
\hline \multirow[b]{2}{*}{ Year } & \multirow[b]{2}{*}{ N hospitals } & \multirow{2}{*}{$\begin{array}{l}\text { N stays } \\
100 \%\end{array}$} & \multicolumn{2}{|c|}{$\begin{array}{l}\text { Stays with } \\
\text { cefazolin, } 2-8 \mathrm{~g} \text {, } \\
\text { no other } \\
\text { antimicrobials }\end{array}$} \\
\hline & & & $\mathbf{N}$ & Per cent \\
\hline 1999 & 110 & 18562 & 9802 & 53 \\
\hline 2000 & 110 & 20559 & 11691 & 57 \\
\hline 2001 & 110 & 21790 & 12597 & 58 \\
\hline 2002 & 110 & 23254 & 13274 & 57 \\
\hline 2003 & 110 & 24606 & 14823 & 60 \\
\hline 2004 & 110 & 25917 & 16942 & 65 \\
\hline 2005 & 110 & 27756 & 18551 & 67 \\
\hline 2006 & 110 & 29267 & 19761 & 68 \\
\hline 2007 & 108 & 30727 & 21336 & 69 \\
\hline 2008 & 107 & 32708 & 22796 & 70 \\
\hline 2009 & 106 & 34256 & 23930 & 70 \\
\hline 2010 & 104 & 35692 & 25264 & 71 \\
\hline
\end{tabular}
prophylaxis, Belgium, 1999-2010

that there is still a lot of unexplained residual variability. Observed and predicted values for the full model, and for hospitals according to the year of funding AMT, are shown in figure 1.

\section{Pneumonia QI}

A total of 327635 hospitals stays between 1999 and 2010 were available for analyses $(95 \%$ of the total number of stays coded APR DRG 139, after exclusions for coding errors). All had 'pneumonia' as an admission diagnosis, so we assume that the vast majority of patients were admitted for community-acquired pneumonia. (In theory, it is possible that some patients were transferred from another hospital with a hospital-acquired pneumonia.) From 1999 to 2010, median LoS in this population decreased from 9 to 7 days, percentile 25 for age decreased from 16 to 11 years. Case fatality decreased in all four categories of SoI, from $1.6 \%$ to $0.4 \%$ in less severe illness (SoI 1), and from $40 \%$ to $33 \%$ in more severe illness (SoI 4).

Trends in the number of hospital stays and the QI are shown in table 2. Between 1999 and 2010, the mean DDD of AM other than J01C decreased from 96 to 64/ 100 days of hospitalisation for pneumonia; the $\mathrm{O} / \mathrm{P}$ ratio (all AM) increased from 0.72 to 0.97 .

Based on LR tests comparing models with and without specific change points, the change point 'year of AMT' was not retained in the models for the DDD QI ( $p=0.4659)$, nor for the $\mathrm{O} / \mathrm{P}$ QI ( $p$ : 0.0997). This means that there was no statistically significant change in slope for these outcomes, that is, the year a hospital received funding for their AMT.

For the DDD QI, the years 2001 and 2006 were identified as statistically significant change points ( $p<0.0001$ and 0.0009 , respectively). In 2001, the QI 


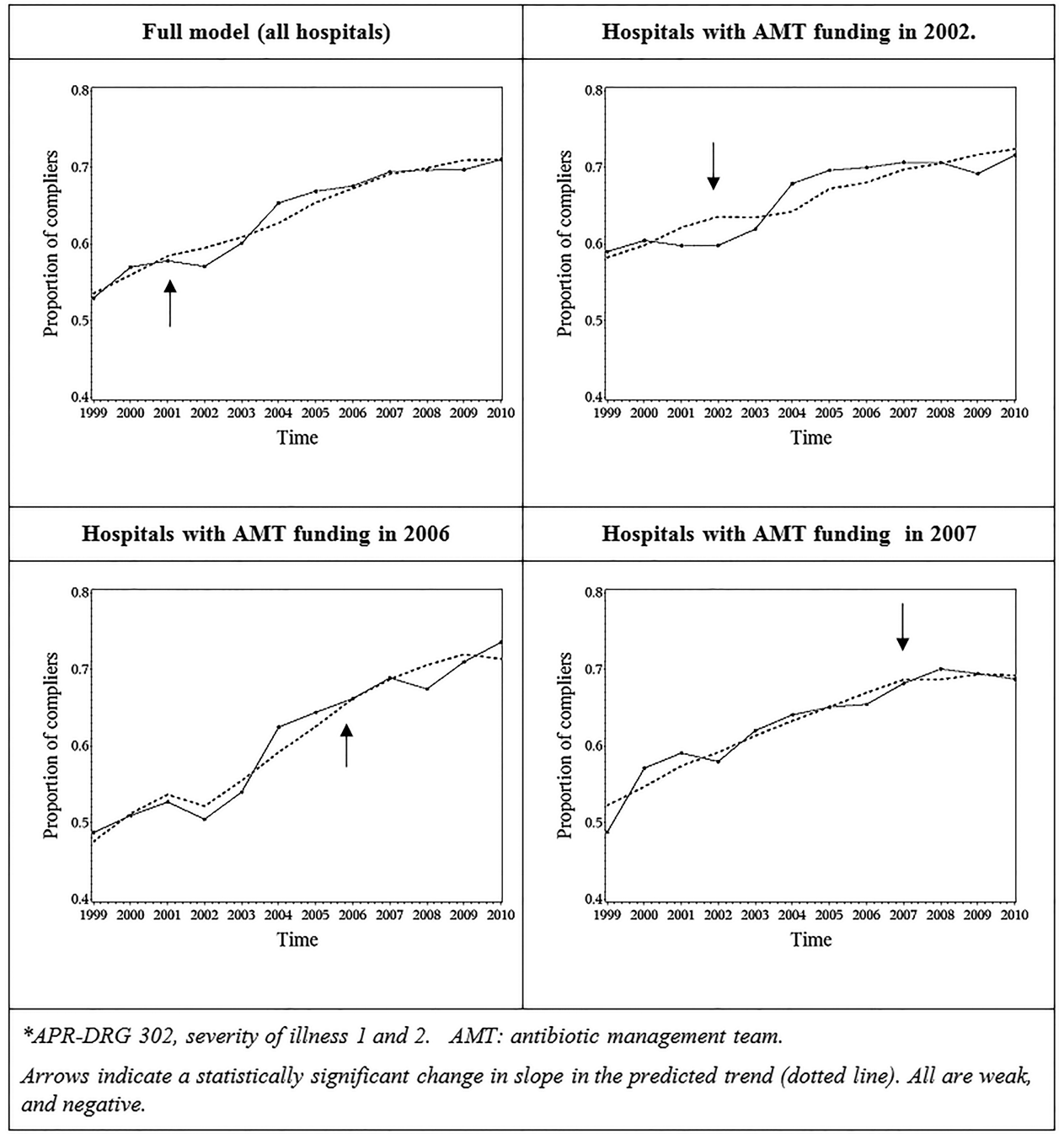

Figure 1 Average observed and predicted (dotted) evolution of compliance with guidelines for surgical antibiotic prophylaxis for lower limb surgery*, Belgium, 1999-2010. AMT, antimicrobial management teams; APR-DRG, all-patient-refined diagnosis-related group.

decreased, and the effect was strong. In 2006, the QI decreased at a slower pace than before. The adjusted $\mathrm{R}^{2}$ for the final model was 0.8072 . For the $\mathrm{O} / \mathrm{P}$ ratio QI, the year 2001 was not retained in the model $(\mathrm{p}=0.9884)$, while the year 2006 was identified as a statistically significant change point $(p=0.0310)$ : the QI increased, but at a slower pace than before. The adjusted $\mathrm{R}^{2}$ for the final model was 0.7559 . For these two pneumonia outcomes, the high adjusted $\mathrm{R}^{2}$ indicates that the models perform well in explaining the variability in the data. The effect of the '2006' change point on both QIs was weak and of doubtful clinical significance, as shown in figure 2.

\section{General features of statistical modelling}

All 3 QIs showed high heterogeneity: baseline values as well as changes over time varied widely across hospitals. Another feature was the complexity of the predicting models, which involved many parameters, and interactions, rendering interpretation sometimes difficult, so that the effect of 'change points' is better assessed visually on the figures presented. Of importance, the introduction of time lags in the models (ie, taking into account the year before or the year after the 'change points') did not improve the models. A complete description of the results, interaction terms and estimates is available in the full study report. ${ }^{18}$

\section{DISCUSSION}

\section{Key findings}

In 1999, 53\% of patients admitted in hospital for major lower limb orthopaedic surgery received the recommended AM for surgical antibioprophylaxis, with a dosage in the expected range, and no other AM. This figure was $71 \%$ in 2010 . For patients treated for pneumonia over the same period, the mean consumption of AM other than penicillin decreased from 96 DDD to 64 DDD/100 hospital-days, and the ratio of oral/parenteral DDD increased from 0.72 to 0.97 . There was high heterogeneity between hospitals. No positive effect could be 
Table 2 Hospital stays for simple pneumonia* and quality indicators for antimicrobial treatment, Belgium, 1999-2010

\begin{tabular}{|c|c|c|c|c|c|c|c|c|}
\hline \multirow[b]{2}{*}{ Year } & \multirow[b]{2}{*}{ N hospitals } & \multirow[b]{2}{*}{ N stays } & \multicolumn{3}{|c|}{ Mean DDD/100 patient-days } & \multicolumn{3}{|c|}{ DDD oral/DDD parenteral } \\
\hline & & & J01C & Other & Total & J01C & Other & Total \\
\hline 1999 & 112 & 22385 & 66 & 96 & 161 & 0.57 & 0.84 & 0.72 \\
\hline 2000 & 112 & 23268 & 67 & 97 & 164 & 0.58 & 0.87 & 0.74 \\
\hline 2001 & 112 & 24165 & 70 & 100 & 170 & 0.62 & 0.91 & 0.77 \\
\hline 2002 & 112 & 25424 & 75 & 97 & 172 & 0.66 & 0.81 & 0.74 \\
\hline 2003 & 112 & 26331 & 83 & 89 & 172 & 0.69 & 0.83 & 0.76 \\
\hline 2004 & 112 & 25201 & 91 & 82 & 172 & 0.79 & 0.82 & 0.80 \\
\hline 2005 & 112 & 30145 & 97 & 81 & 178 & 0.83 & 0.93 & 0.87 \\
\hline 2006 & 112 & 26739 & 99 & 74 & 173 & 0.87 & 0.96 & 0.91 \\
\hline 2007 & 110 & 28872 & 99 & 68 & 167 & 0.89 & 1.04 & 0.95 \\
\hline 2008 & 110 & 29823 & 101 & 65 & 166 & 0.90 & 1.02 & 0.95 \\
\hline 2009 & 109 & 33611 & 102 & 63 & 165 & 0.90 & 1.11 & 0.98 \\
\hline 2010 & 107 & 31671 & 106 & 64 & 170 & 0.88 & 1.12 & 0.97 \\
\hline
\end{tabular}

detected at the national level for these three QIs in relation to the timing of implementation of the policy. In 2001, the decrease in the pneumonia DDD indicator was a statistically and most likely clinically significant deviation from expected trends.

\section{Strengths and limitations}

To the best of our knowledge, this is the first study specifically evaluating a national policy of implementing and funding AM stewardship programme at hospital level. Another country-Scotland-funds AMT in hospitals since $2008,{ }^{19}$ as part of a comprehensive policy to improve
AM prescribing. The Scottish policy as a whole appears to be successful-but the extent to which the funding of AMT contributes to this success remains unknown. ${ }^{20}$

Its major strengths are an exhaustive patient-based database including all Belgian hospitals over 12 years. The fact that the intervention was implemented at different times allowed us to compare QIs both within and between hospitals (hospitals before and after implementation of the policy, as well as hospitals with and without the policy); our fine-tuned case-mix adjustment removed confounding due to differences in the patient population. The two APR-DRG studied account for the largest

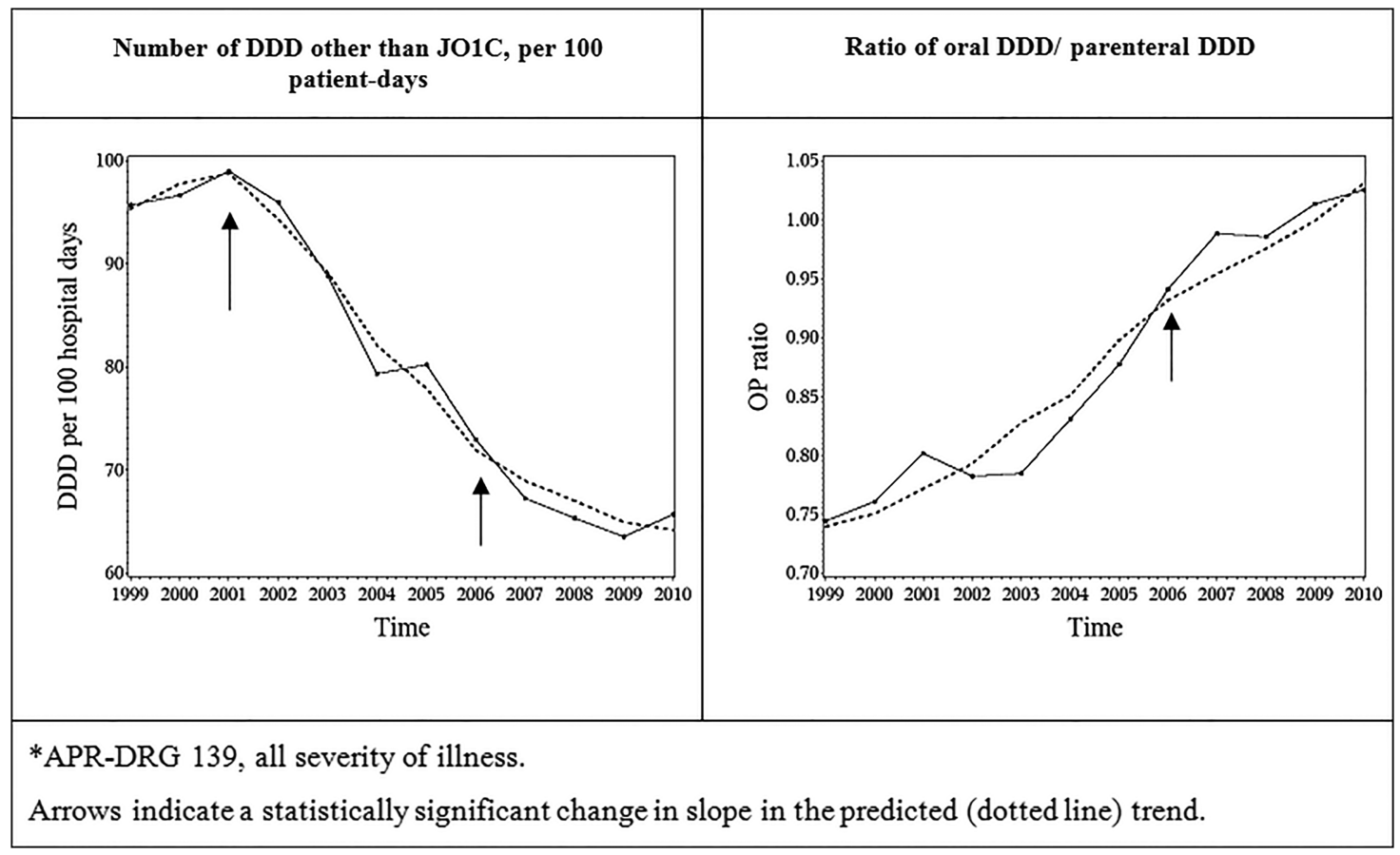

Figure 2 Average observed and predicted (dotted) evolution of quality indicators for antimicrobial treatment of communityacquired pneumonia* in hospitals, Belgium, 1999-2010. APR-DRG, all-patient-refined diagnosis-related group; J01C, ATC code, $\beta$-lactam penicillin; DDD: defined daily dose. 
number of patients receiving AM in hospitals. The trend in improvement for the surgery QI is robust, even if true compliance is less than what we measured, as our QI does not include timing of AM administration. We used state-of-the art statistical methods for modelling the trends of our QI, and acknowledged that change can take time by introducing time lags in the model (taking into account one year before, one year after the 'change point'); nonetheless, these did not improve the model. The major limitation of this study is that we only looked at three QIs for two indications. These QIs do not measure patient outcomes such as surgical site infection or mortality (but these are only indirectly related to appropriate AM prescription, eg, overshooting could also cure a patient). One QI is questionable. The decreasing trends in the consumption of DDD other than penicillin mainly reflect a decrease in cephalosporins and macrolides which offsets an increase in carbapenems and fluoroquinolones. The decrease is also partly explained by a shift towards broad-spectrum penicillin (J01CR, or combinations, such as amoxicillin-clavulanic acid) ${ }^{18}$ It is therefore unclear whether the decrease in this QI indeed represents a true improvement in quality.

\section{Interpretation}

At least two out of three of our QIs describe a true improvement in AM use for patients admitted to hospital for pneumonia and lower limb surgery between 1999 and 2010 in Belgium; these improvements have benefited a very large number of patients. Exactly why this happened is unclear. Although 2001 (a 'change point' for the DDD QI) was the year of a very large awareness campaign for AM, this campaign targeted upper respiratory tract infections in the community.

There are several possible explanations for the lack of association between improvement in the QIs and the year of implementation of AMT in hospitals. The selected items for QI, that is, prophylaxis for major limb orthopaedic surgery and AM therapy in community acquired pneumonia, are items/topics likely to have been addressed in the hospital AM policy at different time points, fully or largely independent from the implementation of AMT. AMT were not given targets, and therefore might have chosen other priorities for interventions than the indications analysed here, despite these being the indications with the highest volume of patients receiving AM. (In 2013, however, all AMT in Belgium conducted audits for antibioprophylaxis in surgery in which serious gaps were identified. ${ }^{21}$ ) It is also possible that the earmarked AMT funding was not always used directly for AMT implementation. A major shortcoming resides in the absence of data on the actual use of funding within hospitals, which may not be easily extracted from the annual AMT activity reports.

\section{Generalisability}

The generalisability of our study finding is limited by the intrinsic complexity of evaluating the quality of AM use in hospitals. We investigated only a limited number of QIs for a limited number of indications: this does not provide a complete picture of trends in quality of AM use in Belgian hospitals, and of the effect of implementing and funding AMT in hospitals. This study should ideally be complemented by other indicators, which might provide different results. For instance, worrying trends are observed for hospital use of some AM; between 1999 and 2010, the use of colistine quadrupled and the use of carbapenem more than doubled (6-24 DDD, and 118-265, per 10000 patient-days, respectively). ${ }^{18}$

Political commitment for tackling the problem of AM resistance in general, and improving AM use in hospitals in particular, has clearly been increasing in the past decade in Europe, North America and elsewhere. $^{722-26}$ Recommendations for strengthening the legal basis and core funding of antibiotic stewardship programmes acknowledge the urgent need for building an evidence base for such policies through good quality research, ${ }^{78}$ and we believe that this study contributes to this objective.

\section{CONCLUSIONS}

Our study has shown improvement on selected QIs for AM use in the treatment of pneumonia, and in antibioprophylaxis for lower limb orthopaedic surgery in Belgian hospitals between 1999 and 2010. These improvements could not be related to a policy of funding AMT. Changes observed may therefore reflect the more multifactorial and continuous change process of quality improvement, of which the implementation and funding of AMT was rather a consequence than a primary driver.

Our findings do not question the need for AMT, nor the need for continuation of AMT funding. Several recommendations could be made in order to make the best of the Belgian unique political, and financial commitment in that field. Transparency in the use of earmarked funding for AMT at hospital level could be improved. Trends of other AM QIs deserve more research. AMTs in hospitals should benefit from more guidance in terms of identifying priorities for action.

\section{Author affiliations}

${ }^{1}$ Healthcare-Associated Infections \& Antimicrobial Resistance, Public Health and Surveillance Department, Scientific Institute of Public Health, Brussels,

Belgium

${ }^{2}$ Interuniversity Institute for Biostatistics and Statistical Bioinformatics (I-BIOSTAT), University of Hasselt, Diepenbeek, Belgium

${ }^{3}$ Microbiology Department, University Hospital, Antwerp, Belgium

${ }^{4}$ Centre for Health Economic Research and Modelling Infectious Diseases

(CHERMID), Vaccine \& Infectious Disease Institute (VAXINFECTIO), University

of Antwerp, Antwerp, Belgium

${ }^{5}$ Internal Medicine Department, University Hospital, Ghent, Belgium

Acknowledgements The authors sincerely thank the following persons for their contribution to this study: (in alphabetical order) Michiel Costers, Anne Ingenbleek, Evelyn Van Gastel, Yves Parmentier and Sofie Vaerenberg. 
Contributors M-LL, NHa, HG and DV designed the study; M-LL, NHa and RB wrote the first draft; RB, NHe and MA conducted the statistical analyses; FN, $\mathrm{BC}, \mathrm{HG}$ and DV revised the manuscript critically for important intellectual content. All authors read and approved the final manuscript

Funding This study was funded by the Scientific Institute of Public Health (internal grant, 99429 Euros, project number W3035.0116.1). Data have been generated as part of the routine work of the Ministry of Health and National Sick Fund. RB acknowledges support from a Methusalem research grant from the Flemish government not specific for this project.

Competing interests None.

Provenance and peer review Not commissioned; externally peer reviewed.

Data sharing statement Legal restrictions apply to these data, which cannot be shared.

Open Access This is an Open Access article distributed in accordance with the Creative Commons Attribution Non Commercial (CC BY-NC 4.0) license, which permits others to distribute, remix, adapt, build upon this work noncommercially, and license their derivative works on different terms, provided the original work is properly cited and the use is non-commercial. See: http:// creativecommons.org/licenses/by-nc/4.0/

\section{REFERENCES}

1. Torjesen I. Antimicrobial resistance presents an "apocalyptic" threat similar to that of climate change, CMO warns. BMJ 2013;346:f1597.

2. European Center for Disease Prevention \& Control. Surveillance of antimicrobial consumption in Europe 2011. Stockholm, 2014. http:// www.ecdc.europa.eu/en/publications/Publications/ antimicrobial-consumption-europe-surveillance-2011.pdf

3. European Centre for Disease Prevention and Control. Point prevalence survey of healthcare-associated infections and antimicrobial use in European acute care hospitals 2011-2012. Stockholm: ECDC, 2013. http://www.ecdc.europa.eu/en/publications/Publications/healthcareassociated-infections-antimicrobial-use-PPS.pdf

4. Magill SS, Edwards JR, Bamberg W, et al. Multistate point-prevalence survey of health care-associated infections. N Engl $J$ Med 2014;370:1198-208.

5. Zarb P, Amadeo B, Muller A, et al. Identification of targets for quality improvement in antimicrobial prescribing: the web-based ESAC Point Prevalence Survey 2009. J Antimicrob Chemother 2011;66:443-9.

6. Davey P, Brown E, Charani E, et al. Interventions to improve antibiotic prescribing practices for hospital inpatients. Cochrane Database Syst Rev 2013;4:CD003543.

7. Allerberger F, Gareis R, Jindrak V, et al. Antibiotic stewardship implementation in the EU: the way forward. Expert Rev Anti Infect Ther 2009;7:1175-83.

8. Society for Healthcare Epidemiology of America, Infectious Diseases Society of America, Pediatric Infectious Diseases Society. Statement on Antimicrobial Stewardship by the Society for Healthcare Epidemiology of America (SHEA), the Infectious Diseases Society of America (IDSA), and the Pediatric Infectious Diseases Society (PIDS). Infect Control Hosp Epidemiol 2012;33(4 Special Topic Issue: Antimicrobial Stewardship):322-7.

9. Goossens H, Coenen S, Costers M, et al. Achievements of the Belgian Antobiotic Policy Coordination Committee (BAPCOC). Euro Surveill 2008;13:pii: 19036
10. Van Gastel E, Costers M, Peetermans WE, et al. Nationwide implementation of antibiotic management teams in Belgian hospitals: a self-reporting survey. J Antimicrob Chemother 2010;65:576-80.

11. 3M information systems. All patient refined diagnosis related groups (APR-DRGs). Version 20.0. Methodology overview. 2003. http:// www.hcupus.ahrq.gov/db/nation/nis/ APRDRGsV20MethodologyOverviewandBibliography.pdf

12. WHO International Working Group for Drug Statistics Methodology, WHO Collaborating Centre for Drugs Statistics Methodology, WHO Collaborating Centre for Drug Utilization Research and Clinical Pharmacological Services. Introduction to drug utilization research. Oslo, Norway, 2003. http://www.who.int/medicines/areas/quality_ safety/safety_efficacy/Drug\%20utilization\%20research.pdf

13. The Sanford guide to antimicrobial therapy. 23rd edition of the Belgian/Luxemburg Version-2012-2013. 2012

14. Dellit TH, Owens RC, Mcgowan JE, et al. Infectious Diseases Society of America and the Society for Healthcare Epidemiology of America guidelines for developing an institutional program to enhance antimicrobial stewardship. Clin Infect Dis 2007:44:159-77.

15. Rhew DC, Tu GS, Ofman J, et al. Early switch and early discharge strategies in patients with community-acquired pneumonia: a meta-analysis. Arch Intern Med 2001:161:722-7.

16. Minalu G, Aerts M, Coenen S, et al. Adaptive change-point mixed models applied to data on outpatient tetracycline use in Europe. Stat Model 2013;13:253-74.

17. Molenberghs G, Verbeke G. Models for discrete longitudinal data. Springer, 2005.

18. Lambert M, Hammami N, Catry B, et al. Antimicrobial consumption in Belgian hospitals for selected diagnoses, 1999-2010, and association with policies aimed at promoting rational use. Brussels 2014D/2014/2505/07. https://www.wiv-isp.be/nsih/download/Home/ AMTABU_FINAL_REPORT.pdf

19. Scottish Health Department. Prudent Antimicrobial Prescribing: the Scottish Action Plan for managing antibiotic resistance and reducing antibiotic related Clostridium difficile associated disease. 200830. http://www.scottishmedicines.org.uk/files/sapg/CEL_30_2008_-Antimicrobial_Prescribing.pdf

20. Nathwani D, Sneddon J, Patton A, et al. Antimicrobial stewardship in Scotland: impact of a national programme. Antimicrob Resist Infect Control 2012;1:7.

21. Van Gastel E. Antibioprophylaxis in surgery: results of internal audits. Brussels, Belgium, 2014. http://www.health.belgium.be/ eportal/Myhealth/Properuse/Antibiotics/Healthcarefacilities/index.htm

22. Australian Commission on Safety and Quality in Healthcare. Antimicrobial stewardship in Australian hospitals. 2011. http://www. theasm.org.au/assets/ASM/44471-Antimicrobial-Stewardship20111.pdf

23. Mount Sinai Hospital. Antimicrobial Stewardship Program: Strategic Plan 2013-2016. Toronto, Canada: University Health Network, 2013. http://www.antimicrobialstewardship.com/sites/default/files/ 2013-2016_strategic_plan.pdf

24. Laxminarayan R, Duse A, Wattal C, et al. Antibiotic resistance-the need for global solutions. Lancet Infect Dis 2013;13:1057-98.

25. Ashiru-Oredope D, Sharland M, Charani E, et al. Improving the quality of antibiotic prescribing in the NHS by developing a new Antimicrobial Stewardship Programme: Start Smart-Then Focus. $J$ Antimicrob Chemother 2012;67(Suppl 1):I51-63.

26. Trivedi KK, Rosenberg J. The state of antimicrobial stewardship programs in California. Infect Control Hosp Epidemiol 2013;34:379-84. 